\title{
AUDET, Louis-Philippe, docteur en pédagogie, Le système scolaire de la Province de Québec, tome V, Les écoles élémentaires dans le Bas-Canada, 1800-1836. Les éditions de l'Érable, Québec, Canada, MCMLV, XVII-325 p.; tome VI, La situation scolaire à la veille de l'Union, 1836-1840, même édition, MCMLVI, XVIII - 353 p.
}

\section{Lionel Groulx}

Volume 10, numéro 2, septembre 1956

URI : https://id.erudit.org/iderudit/301766ar

DOI : https://doi.org/10.7202/301766ar

Aller au sommaire du numéro

Éditeur(s)

Institut d'histoire de l'Amérique française

ISSN

0035-2357 (imprimé)

1492-1383 (numérique)

Découvrir la revue

Citer ce compte rendu

Groulx, L. (1956). Compte rendu de [AUDET, Louis-Philippe, docteur en pédagogie, Le système scolaire de la Province de Québec, tome V, Les écoles élémentaires dans le Bas-Canada, 1800-1836. Les éditions de l'Érable, Québec, Canada, MCMLV, XVII-325 p.; tome VI, La situation scolaire à la veille de

l'Union, 1836-1840, même édition, MCMLVI, XVIII - 353 p.] Revue d'histoire de l'Amérique française, 10(2), 279-285. https://doi.org/10.7202/301766ar d'utilisation que vous pouvez consulter en ligne. 


\section{LIVRES ET REVUES}

AUDET, Louis-Philippe, docteur en pédagogie, Le système scolaire de la Province de Québec, tome V, Les écoles élémentaires dans le Bas-Canada, 1800-1836. Les éditions de l'Erable, Québec, Canada, MCMLV, XVII-325 pages; tome VI, La situation scolaire à la veille de l'Union, 1836-1840, même édition, MCMLVI, XVIII - 353 pages.

M. Audet a entrepris d'écrire l'histoire du Système scolaire de la Province de Québec. Il en est à son sixième volume. Et il atteint tout juste la date de 1840. C'est dire quelle somme il nous prépare. Oeuvre considérable qui nous révélera un aspect important de l'histoire de la province de Québec, s'il est vrai que l'enseignement public n'est que l'un de ces multiples miroirs où un peuple reflète son image. Un système scolaire, ses structures, ses inspirations, ses idéaux, ce sont là conceptions de l'esprit, conceptions qui procèdent elles-mêmes d'une conception de l'homme et d'une philosophie de la vie. Quel document peut dépasser en valeur celui-là ? Et il faut aussi tenir compte que l'historien ne saurait écrire un tel chapitre sans se reporter constamment - et nous le voyons bien en lisant M. Audet - à tous les éléments de l'histoire intégrale, tant il y a, de part et d'autre, mutuel éclairage.

Dans la Province de Québec ou du Bas-Canada, l'histoire scolaire offre, en outre, à qui l'aborde, cet aspect original ou singulier qu'elle n'y est pas, comme dans les pays indépendants ou adultes et de composition homogène, une efflorescence ou une poussée naturelle, fruit d'un génie ethnique se développant en droiture. Elle est partie d'histoire d'un peuple colonial qui a passé par l'épreuve d'une conquête et par conséquent histoire d'une vie intellectuelle inclinée à des courbes dangereuses, contrainte, en tout cas, à d'incessantes et laborieuses adaptations. Cet aspect-là, non plus, n'a pas échappé à $M$. Audet, même s'il lui arrive, pour la période de 1792 à 1840, par exemple, de nous pré- 
senter un résumé d'histoire générale d'une simplification excessive et d'un bloc trop à part. Pourquoi pas plutôt une histoire parallèle à celle de l'enseignement, où entre les ordres de faits, les influences réciproques nous fussent mieux apparues?

Tout n'est pas neuf en cette histoire d'un système scolaire. L'auteur a largement emprunté à ses prédécesseurs. Et il leur en donne loyalement crédit. Le nouveau, on le trouvera dans la substance documentaire de l'oeuvre. M. Audet abuse peut-être de ce côté-là. Art et histoire ne se repoussent point. L'édifice une fois terminé, on aime l'apercevoir, débarrassé de ses échafaudages, en la pureté de ses lignes architecturales. Par souci, sans doute, d'excessive probité, l'historien n'a rien voulu démolir en son chantier de construction. Au lieu d'être incorporé, fondu dans le texte, le document est souvent juxtaposé, ajouté, quelle qu'en soit la dimension. Profit mince pour le spécialiste qui ne se dispense jamais de se reporter aux sources, à l'original. Profit peut-être pour le commun des lecteurs, dispensés de fastidieuses recherches. Au surplus l'ouvrage se revêt d'un bout à l'autre d'un rigoureux appareil didactique: sommaires ou schémas au début des chapitres, disposition typographique, soulignements des divisions et subdivisions, tableaux de statistiques. M. Audet n'est pas pour rien docteur en pédagogie. Sans doute at-il voulu que chacun de ses lecteurs en fût persuadé.

Le neuf, en cet ouvrage, on le trouvera encore dans l'analyse de la législation scolaire du Bas-Canada. Chacune des lois de l'époque, surtout celle de 1824 (Ecoles de fabrique) et celle de 1829 , passent à leur tour sur la table de dissection. Dissection un peu sèche, qu'on voudrait plus éclairée par l'esprit de l'époque, par le mouvement des idées qui pèsent si fort sur les parlements démocratiques. Mais dissection de main experte et qui marque bien les origines et l'évolution d'un système scolaire en voie de s'édifier. Et puisque nous en sommes à souligner l'apport du nouveau, en cette œuvre, notons en particulier le chapitre sur les "Ecoles normales». Chapitre incontestablement le plus documenté et le mieux élaboré de ces deux volumes. 
Le nouveau, dirons-nous qu'on le trouverait encore en quelques façons de voir ou certains jugements de l'auteur ? Il n'a guère le culte du conformisme. Sur maints points et quelques-uns d'assez de gravité, il s'inscrit résolument en dissidence avec les jugements reçus ou les opinions traditionnelles. Légitime disposition d'esprit, à la seule condition de respecter les faits et tout ce qui s'appelle en histoire objectivité et saine critique. Par exemple, M. Audet, on le sent en maints endroits de ses livres, ne pardonne qu'avec peine aux chefs religieux de l'époque, surtout à Mgr Lartigue, leur intransigeance à l'égard de l'Institution royale et de la loi de 1829. Cette sévérité serait-elle de tout point justifiable ? Aurait-il échappé à l'historien qu'en s'opposant, quoique d'une manière inflexible parfois, à toute immixtion de la politique ou de l'Etat dans l'administration scolaire, comme à toute administration mixte selon la foi, ces évêques, sans trop le savoir, frayaient la voie à la loi de 1846 puis à celle de 1875 ? Mérite de quelque valeur pourtant que d'avoir préparé ces lois de haute sagesse qui allaient émanciper l'école des tutelles étatistes et faire triompher, du même coup, le principe de la confessionnalité. Double gain qui après tout réaliserait, dans la province, un idéal de liberté scolaire à peu près unique au monde. Pour quels motifs en effet, les évêques d'alors se sont-ils opposés tenacement à l'Institution royale, même amendée, si ce n'est qu'ils y voyaient une institution d'Etat sur laquelle les catholiques manqueraient encore de prises suffisantes ? Les amendements ne venaient-ils pas d'ailleurs trop tard au moment où d'autres législations pouvaient offrir plus de garanties ? Si les mêmes évêques ont paru bouder un temps la loi de 1829, la raison n'en serait-elle pas encore qu'elle faisait part trop large à l'influence politique et qu'en un pays catholique, elle excluait presque l'Eglise de l'école ? M. Audet (tome V, p. 116, 136, 137, 160) fait grand état de l'amendement apporté à cette loi en 1830 où le curé de paroisse, même non propriétaire dans l'arrondissement, devenait éligible, cette fois, aux fonctions de syndic. La concession en valait-elle véritablement la peine quand la loi ne contenait aucune stipulation relative à la foi religieuse des syndics, nul dispositif en faveur de quelque enseignement religieux ou du choix des livres de classe ? Du 
reste, les véritables amendements apportés à la loi de 1829, M. Audet ne saurait l'ignorer, ne sont pas de 1830 , mais seulement de 1832. C'est trois ans après la mise en pratique de la législation de 1829 que l'on concède aux curés le droit de visite dans les écoles et un mot à dire dans l'attribution du certificat de compétence et de moralité à l'instituteur. Mais même alors, le clergé avait-il raison de tant se réjouir d'une législation qui ne se montrait guère plus libérale à son sujet que l'Institution royale?

A la vérité, il paraîtra excessif qu'on reproche à des évêques catholiques de s'être opposés à toute forme de neutralité religieuse et d'avoir voulu garder à l'école publique le caractère confessionnel dans une province en grande majorité catholique. Précisément sur une question aussi grave que celle de la confessionnalité scolaire, quoi de plus explicable à l'époque que les intransigeances épiscopales. Quel étrange état d'esprit s'était insinué dans le troupeau des fidèles. Des catholiques (voir mon Enseignement français, tome I, p. 151-152) tolèrent ou soutiennent des écoles neutres ou mixtes selon la foi. Quelques prêtres font de même. Des parlementaires catholiques s'opposent à l'immixtion du clergé dans l'enseignement public. M. Audet s'en rendra meilleur compte lorsqu'il abordera la période d'après 1840 : non seulement un LaFontaine, mais un dévot comme Augustin-Norbert Morin, accordent publiquement leurs préférences à l'école neutre. Pardonnons alors aux autorités religieuses quelques excès de clairvoyance qui nous paraissent moins graves que le péché d'aveuglement. Au reste, M. Audet n'a pas négligé de le noter, mais il aurait pu y insister davantage: l'intransigeance des évêques n'a jamais revêtu forme négative. Ils n'ont jamais été contre quoi que ce soit, sans être pour quelque chose, je veux dire, pour la promotion de l'enseignement et d'abord pour un système scolaire plus approprié aux exigences de leur peuple. Ils n'ont jamais, non plus, réclamé de droits ni de libertés qu'ils ne les aient en même temps consentis à la minorité protestante. Témoignage de générosité et de largeur d'esprit que ne leur a pas refusé lord Durham.

J'ai peur, - le dirai-je aussi ? - que M. Audet n'ait jugé trop sévèrement ce qu'il appelle l'apathie du clergé dans l'éta- 
blissement des écoles de fabrique. Nous ne croyons pas d'abord que l'on puisse tenir la loi de 1824 pour un fiasco. Et sans ignorer certains blâmes épiscopaux à l'adresse de curés négligents, faut-il négliger certaines circonstances telles que celles-ci: rareté des fabriques riches, dimensions géantes des paroisses, manque de prêtres, un grand nombre d'entre eux succombant prématurément aux fatigues d'un ministère épuisant ? Et je prie que l'on ne voit point là plaidoyer complaisant d'un historien ecclésiastique. Avec autant de complaisance, je disculperai le petit peuple du Bas-Canada du reproche d'apathie que lui adresse M. Audet. Que l'auteur n'a-t-il insisté davantage sur les causes historiques de cette apathie ? La fondation de l'Institution royale date de 1801. Mais l'œuvre attendra presque vingt ans avant de se mettre véritablement en opération. Et voilà donc, dans la vie d'un peuple, quatre-vingts ans écoulés depuis 1760 où l'on n'a su mettre à sa portée un système d'enseignement public. Et même en 1801, quel système sujet à caution et par trop justement controversé ! Pourquoi voudrait-on qu'après ces quatrevingt ans d'analphabétisme forcé, une large partie de la population française du Bas-Canada ait encore gardé un goût vif de l'instruction ? Pourtant, c'est dans ce même Bas-Canada, et cela encore M. Audet aurait pu le souligner davantage, qu'en 1821 l'on vient tout près d'organiser une vaste pétition, la première des grandes pétitions populaires de l'époque, pour arracher au gouvernement britannique la sanction d'une loi scolaire depuis trop longtemps différée. Et que dire du réveil, je dirais même du sursaut d'après 1829 , date de la première loi des écoles élémentaires enfin mise à l'épreuve ? Dans la diffusion rapide des écoles, M. Audet voit avec raison un "miracle incroyable» (tome V, p. 137). Et, sans doute, ces écoles sont des écoles subventionnées par l'autorité politique. Mais M. Audet n'a pas manqué de relever non plus en 1834 (p. 248) la disposition des parents à se charger volontairement du soutien de leurs écoles, disposition, il ne l'ignore point, qu'on retrouve déjà sous le Régime français.

Un coup d'œil rapide maintenant sur le sixième tome de l'auteur. Au risque de paraître grincheux, j'aurais envie de 
chicaner M. Audet sur la division de ce tome. Il l'a divisé en quatre parties: I: Situation scolaire à la veille de l'Union; II, Lois des Ecoles normales; III, Sociétés d'éducation et éducateurs ; IV, Enquête Buller. Tout en s'intégrant la $3^{\mathrm{e}}$ et même la $2^{\circ}$, la première partie n'aurait-elle pu prendre place en quatrième lieu ? Avec 1840, nous sommes à la fin d'une époque et à la veille d'une autre. En bonne logique, n'était-ce pas le lieu précis où nous présenter le bilan des derniers accomplissements et aussi les inquiétudes soulevées par l'enquête Buller ?

Cette enquête d'Arthur Buller sur l'enseignement public faisait partie, comme l'on sait, de l'enquête générale de lord Durham. Pour cette période de l'histoire canadienne, M. Audet l'avoue franchement, il a surtout suivi Chester New (Lord Durham, Oxford, 1929.) Information trop restreinte par quoi l'auteur s'exposait à surfaire le lord enquêteur et, par contagion, l'un de ses enquêteurs subalternes: Arthur Buller. La mode est aujourd'hui de réhabiliter lord Durham: on ne lui refuse rien, pas même le génie politique. On le range parmi les constructeurs de l'empire britannique moderne et aussi du Canada de 1867 et d'aujourd'hui. Et c'est tout juste si l'on n'invite pas les Canadiens français à lui dresser une statue. Convient-il d'enjamber si lestement certains faits d'histoire ? Lord Durham, dont nous ne contesterons ni le talent ni certaines vues en avance sur ses contemporains d'Angleterre, n'a pas inventé, que nous sachions, la formule de l'autonomie coloniale ou de ce que l'on appelait alors le «gouvernement responsable ». La formule appartient de plein droit aux réformistes ou libéraux des provinces britanniques de l'Amérique du Nord, même à ceux du Bas-Canada qui l'ont préconisée, explicitée bien avant 1838. Le mérite de lord Durham, et ce mérite n'est pas mince, fut de proposer, avec force, cette formule d'avenir au gouvernement et à l'opinion britannique qui n'y comprenaient rien. Et ce fut encore de voir clair, et l'un des premiers, dans l'imbroglio politique des colonies anglaises de l'Amérique du Nord. Mais là s'arrête le rôle de l'enquêteur. Il n'a pas non plus inventé la formule du fédéralisme d'où serait sorti le «grand Canada». Elle était dans l'air bien avant lui. Précisément, en avril 1838, Joseph Howe la préconisait au parlement d'Halifax. En mai de la même année le journal 
Nova Scotian élaborait ce que l'on appellera: a perfect epitome of the Quebec scheme. Il serait bon de se rappeler, au surplus, que Durham était moins fédéraliste que partisan d'un Etat unitaire, ne concevant d'ailleurs l'Etat fédéral pour les CanadasUnis, que comme un acheminement à l'«Union législative ».

Je ne comprends pas non plus que M. Audet se soit laissé emballer par le rapport d'Arthur Buller sur l'éducation. Que contenait, en effet, de si original l'œuvre du jeune enquêteur ? Luimême l'a avoué très ingénûment: "Je n'ai fait nul effort d'originalité ». Au vrai, il a à peu près tout emprunté au système d'écoles nationales d'Irlande, quand ce n'était pas au système scolaire américain, ou encore au système scolaire prussien alors très haut coté en Angleterre, surtout parmi les radicaux. Il est de même fort possible que l'enquêteur ait pu connaître le système prussien par un rapport de Victor Cousin traduit en anglais dès 1834 et très lu dans le royaume. Quant aux réformes de structure proposées par le jeune Buller dans le système scolaire du Bas-Canada, ces réformes, M. Audet aurait pu se rappeler, puisque la chose ne lui a pas échappé, que les Rapports du Comité permanent de l'Education, comité institué par l'Assemblée législative du Bas-Canada, les avait déjà presque toutes proposées.

Je ne voudrais point, par ces quelques observations, amoindrir l'ampleur de l'œuvre entreprise par M. Audet et déjà menée, avec un appréciable succès jusqu'au sixième tome. Entreprise d'historien qui mérite tout éloge. L'auteur ne néglige aucune peine pour pousser ses recherches le plus loin possible. Il fait preuve d'esprit critique. On voudrait peut-être, à sa façon d'écrire, un peu plus d'élégance. On ne saurait lui reprocher de manquer à la sobriété. Puisse-t-il achever son œuvre. On aperçoit ce que tout un siècle d'histoire encore à parcourir lui imposera de recherches et d'études, surtout à mesure que s'accroîtra la complexité des problèmes. Il faut souhaiter bon voyage aux travailleurs de l'esprit qui osent se jeter en pareilles aventures. Et le profit sera grand pour les prochaines synthèses historiques.

Lionel GroulX, ptre 